\title{
THE ARCONS PIPELINE: DATA REDUCTION FOR MKID ARRAYS
}

\author{
J. C. van Eyken ${ }^{1,2,3}$, M. J. Strader ${ }^{1}$, A. B. Walter $^{1}$, S. R. Meeker ${ }^{1}$, P. Szypryt $^{1}$, C. Stoughton ${ }^{4}$, K. O’Brien ${ }^{5}$, D. Marsden ${ }^{1}$, \\ N. K. RicE ${ }^{1}$, Y. LIN $^{1}$, AND B. A. MAZIN ${ }^{1}$ \\ ${ }^{1}$ Department of Physics, UC Santa Barbara, Santa Barbara, CA 93106, USA; vaneyken@ipac.caltech.edu \\ ${ }^{2}$ Las Cumbres Observatory Global Telescope Network, 6740 Cortona Drive, Suite 102, Goleta, CA 93117, USA \\ ${ }^{3}$ Now at NASA Exoplanet Science Institute, California Institute of Technology, 770 South Wilson Avenue, M/S 100-22, Pasadena, CA 91125, USA \\ ${ }^{4}$ Fermilab Center for Particle Astrophysics, Batavia, IL 60510, USA \\ ${ }^{5}$ Department of Physics, University of Oxford, Denys Wilkinson Building, Keble Road, Oxford, OX1 3RH, UK \\ Received 2014 November 26; accepted 2015 June 18; published 2015 July 27
}

\begin{abstract}
The Array Camera for Optical to Near-IR Spectrophotometry, or ARCONS, is a camera based on Microwave Kinetic Inductance Detectors (MKIDs), a new technology that has the potential for broad application in astronomy. Using an array of MKIDs, the instrument is able to produce time-resolved imaging and low-resolution spectroscopy constructed from detections of individual photons. The arrival time and energy of each photon are recorded in a manner similar to X-ray calorimetry, but at higher photon fluxes. The technique works over a very large wavelength range, is free from fundamental read noise and dark-current limitations, and provides microsecond-level timing resolution. Since the instrument reads out all pixels continuously while exposing, there is no loss of active exposure time to readout. The technology requires a different approach to data reduction compared to conventional CCDs. We outline here the prototype data reduction pipeline developed for ARCONS, though many of the principles are also more broadly applicable to energy-resolved photon counting arrays (e.g., transition edge sensors, superconducting tunnel junctions). We describe the pipeline's current status, and the algorithms and techniques employed in taking data from the arrival of photons at the MKID array to the production of images, spectra, and time-resolved light curves.
\end{abstract}

Key words: instrumentation: detectors - methods: data analysis

\section{INTRODUCTION}

The recent development of superconducting Microwave Kinetic Inductance Detectors (MKIDs; Day et al. 2003; Mazin et al. 2012) opens the door to a promising new generation of UV-to-near-IR astronomical imaging instruments. By constructing an array of MKIDs, it is possible to build a detector analogous to a conventional CCD that is also able to resolve individual photons, recording their arrival times and energies in a manner similar to X-ray microcalorimeters. It is therefore possible to obtain time-resolved imaging and spectroscopy in a single observation. The wavelength range can be very large, potentially as much as $\approx 0.1-5 \mu \mathrm{m}$ (in comparison to $\approx 0.3-1 \mu \mathrm{m}$ for CCDs), with a theoretical energy resolution limit as high as $R=E / \Delta E=\lambda / \Delta \lambda \sim 100$ for a $100 \mathrm{mK}$ operating temperature, and with microsecond time resolution. The devices do not suffer from the intrinsic readout-noise and dark-current issues inherent to traditional CCDs, and as the pixels can be read out simultaneously and continuously, there is no loss of on-sky exposure time during readout. Data can be read and processed in real-time, potentially allowing for live science-image telescope guiding and flux monitoring with no loss of throughput, as well as focal-plane speckle control for high-contrast imaging (Martinache et al. 2014, S. R. Meeker et al., 2015 in preparation).

The Array Camera for Optical to Near-IR Spectrophotometry (ARCONS; see Mazin et al. 2013) is a prototype MKID instrument which has now been tested in the field at both the Lick 120" and Palomar 200" telescopes. The current Palomar design consists of a 2024 pixel $(44 \times 46)$ MKID array, with a median spectral resolution $R \approx 6.5$ at $4000 \AA$, and approximately $70 \%$ useable pixel yield. The instrument is sensitive across a bandwidth of $\approx 4000-11000 \AA$, constrained only by the optics passband. Since the technology is still in its infancy, we expect to make significant improvement in the specifications with time. Nevertheless, the first trial telescope runs have already yielded preliminary science results, including the suggestion of optical enhancements in early giant pulses from the Crab Pulsar (Strader et al. 2013), and the detection of orbital expansion in the AM CVn binary system, SDSS J0926+3624 (Szypryt et al. 2014).

A detailed description of ARCONS is given by Mazin et al. (2013). Here we describe the prototype data reduction process developed to handle the ARCONS data. The current development version of the software can be found online at https:// github.com/bmazin/ARCONS-pipeline. The data reduction pipeline is a work in progress, and much refinement is expected as the MKID technology matures; the basic components are in place, however, to produce images, spectroscopy, and timing analyses.

Section 2 provides a brief overview of the main sections of the pipeline and describes the main data products. Section 3 describes the firmware algorithms used to produce the raw data output from the camera. Section 4 describes each of the steps in the data reduction and calibration in detail, from the initial photon detection through to fully calibrated photon event lists, and in the process touches on some characterization of the current ARCONS instrument. Section 5 describes the process of obtaining three main science data products: images, spectroscopy, and photometry. A brief summary follows in Section 6.

\section{OUTLINE}

The ARCONS pipeline is written in Python, and makes extensive use of the fast array handling and vectorization 
capabilities provided by the NumPy and SciPy packages (Oliphant 2007), ${ }^{6}$ along with astronomical tools from the Astropy package (Astropy Collaboration, et al. 2013), ${ }^{7}$ and the data visualization tools from the Matplotlib package (Hunter 2007). Owing to the large quantities of data to be handled, some care has been taken to optimize the various processing algorithms for speed and efficient disk access, though there remains room for further optimization. The PyTables library ${ }^{8}$ provides an interface to the Hierarchical Data Format (HDF5) standard for file handling, ${ }^{9}$ which is the primary data storage format used in the pipeline. The HDF5 standard allows for versatile structuring of very large data sets and provides various tools for efficient manipulation of on-disk data.

The full reduction process can be divided into three broad steps: the creation of the raw data read from the camera; reduction of the data to produce fully calibrated and flagged photon-event lists; and the creation of the final data output products (images, spectra, or light curves) from those photon lists.

The continuous monitoring and readout of each pixel occurs largely in firmware (Section 3). The raw data are saved in HDF5-format, as are the various intermediate data products created later in the pipeline. To keep file sizes manageable, raw data are written to a new file every five minutes, yielding typical file sizes of a few hundred $\mathrm{Mb}$ to $\sim 1 \mathrm{~Gb}$ for our instrument configuration (depending on the total flux observed). We refer to these as "observation files." Various metadata are also recorded in the files, including the beammap used to associate each MKID resonator with the correct pixel location in the array (see Section 3.4), telescope pointing and status information, and the precise start time of the data in the file.

A number of steps are performed to calibrate the data. Cosmic rays can be cleaned (Section 4.1), dead or unassigned pixels are masked, and intermittent "hot" pixels are identified (Section 4.2). Exposures of laser reference sources are used to calibrate the mapping of resonator phase-shift to wavelength for each photon (Section 4.3). Twilight sky exposures are used to normalize the responses of the pixels with respect to each other as a function of wavelength (Section 4.4). An overall spectral response calibration is then applied by observing a known spectral standard, in order to flatten the effective wavelength response of the instrument (Section 4.5). Where necessary, nonlinearity due to high count rates is accounted for (Section 4.6). Finally, astrometric calibrations are performed to identify the precise location of each detector pixel on the sky at a given time, and hence assign sky coordinates to each detected photon (Section 4.7). Most of these steps involve the creation of a small intermediate calibration file that is used to apply corrections to one or more raw observation files.

The result of this processing is a calibrated photon list for every raw data file (Section 4.8). The associated per-pixel exposure time mask is also stored in the same file, as well as a copy of all calibration files applied, in order to keep an easily traceable audit trail for diagnostic purposes.

\footnotetext{
6 Eric Jones, Travis Oliphant, Pearu Peterson and others. SciPy: Open Source Scientific Tools for Python, 2001- http://www.scipy.org/.

7 http://www.astropy.org

8 Francesc Alted, Ivan Vilata, and others, PyTables: Hierarchical Data sets in Python, 2002 - http://www.pytables.org.

9 The HDF Group. Hierarchical data format version 5, 2000-2010. http:// www.hdfgroup.org/HDF5.
}

With the photon lists in hand, we can produce several science products. For imaging, the photon lists can be read into a module which stacks the photons onto a virtual pixel grid, using the exposure time masks to weight the photons according to the effective total exposure time in each virtual pixel (Section 5.1). The wavelength range can optionally be restricted to create images in different bands, which, if desired, can then be combined to create color images.

Alternatively, time-resolved photometry can be performed similarly to traditional CCD photometry (Section 5.2). If the effective integration times required are short, this can be performed on the calibrated data without running it first through the full image stacking module. If longer integration times are desired, it is possible to run photometry on the stacked and de-rotated images. Again, the photometry can be restricted in wavelength to emulate photometric filtering, allowing for simultaneous multi-band photometry where desired. Where high timing precision is required, photon arrival times can be corrected to Solar System barycentric time.

If spectra are required (Section 5.3), the photons for a chosen aperture can similarly be extracted, with or without prior image stacking, and the energy plotted as a function of wavelength integrated over a given effective exposure time (analogous to Integral Field Spectroscopy).

The ultimate data product (currently partially implemented) will be a four-dimensional data "hypercube," essentially a fourdimensional histogram of photon events with dimensions of R.A., decl., wavelength, and time. We describe each of the steps in the current pipeline in more detail below.

\section{ARCONS FIRMWARE: CREATING THE RAW DATA}

\subsection{Pulse Detection}

The firmware for the digital readout for ARCONS is described in detail by McHugh et al. (2012). Each pixel comprises a small superconducting resonant circuit (a single MKID) tuned to a unique resonant frequency. A microlens array with a $92 \%$ fill factor focuses the light onto the inductor part of the pixel. When a photon is absorbed in the inductor, the inductance of the circuit, and therefore its resonant frequency, briefly changes. Each resonator is driven by a microwave probe tone, the phase of which will change with the pixel's resonant frequency. The firmware detects the absorption of photons as a sudden jump in the phase of a pixel's probe tone, followed by an exponential decay back to the baseline phase (Figure 1). The phase timestream from each pixel is passed through a matched filter made with a template phase pulse specific to the pixel. The firmware also keeps track of the baseline of the phase for each pixel with an infinite impulse response (IIR) low-pass filter. When a peak is detected above a threshold value, the firmware generates a photon data packet to be saved to disk. By design, a $100 \mu$ s dead time $(10 \mu$ s in the most recent ARCONS implementation) follows each photon detection, during which no other photon events will be recorded to disk. This is to prevent false photon detections from phase noise during the decay back to the baseline. The effect of this dead time on the linearity of the pixel's response is corrected (See Section 4.6). The phase timestream itself is not saved while observing due to the prohibitively high storage and datatransfer rate requirements. 


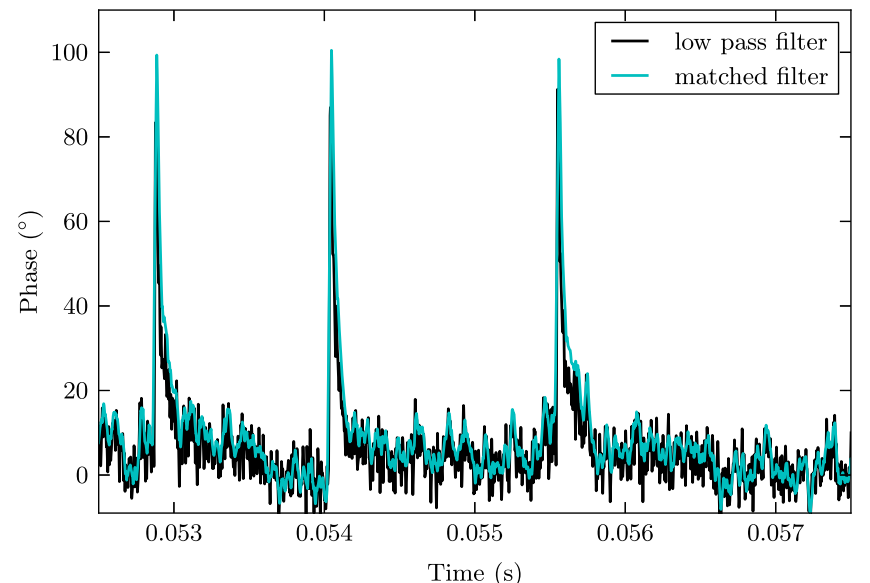

Figure 1. Three photon pulses as seen in the phase timestream from one MKID, where the phase has passed through a low-pass filter with a corner frequency at $250 \mathrm{kHz}$ (black line) or a matched filter made with an average pulse template (cyan line).

\subsection{Raw Data Output}

The raw photon data packets are stored in an HDF5 file, organized by readout circuit board and pixel (Figure 2), with a single data table allocated for each pixel. Each row of a pixel's data table contains a list of 64 bit packets representing the photons detected in one second of exposure. The packet records the pixel where the photon landed, the phase value at the peak caused by the photon in radians, the concurrent phase baseline, and the arrival time of the photon relative to the beginning of the current second (Figure 3).

\subsection{Barycentric Photon Arrival Times}

To determine a photon's arrival time, first the start time of the observation is extracted from the raw HDF5 data file's header. Initially this is formatted as a Unix timestamp, but it is later converted to UTC or to a Julian Date. The 20 bit timestamp in a 64 bit photon packet is the number of output sample time steps since the beginning of the second, where a "time step" is currently $1 \mu \mathrm{s}$. The second in which the photon arrives relative to the start time of the observation corresponds to the row number in the pixel data table where it is stored. The complete absolute time is found by adding the start time of the observation, the seconds elapsed since the beginning of the observation, and the photon timestamp in $\mu \mathrm{s}$. The timestamps are then corrected for a $41 \mu$ s delay in the firmware (previously measured by illuminating the detector with a light emitting diode triggered by the pulse-per-second signal from our GPS clock reference).

In addition, there are occasions when a readout board is slow to offload its data to disk on completion of integration for an observation file, and may be delayed at this point while the other boards have begun collecting data for the next file. In this case, the slow board will start taking data for the new file exactly one second later than the others (readout boards always start recording data at the beginning of a second). The timestamps from this board require a $1 \mathrm{~s}$ offset, since the start time for this board is one second off from the start time read from the header. Log files written during observation indicate when these second offsets are needed. There is an additional problem in that the slow board therefore misses a second of data that the others have recorded. To compensate, we

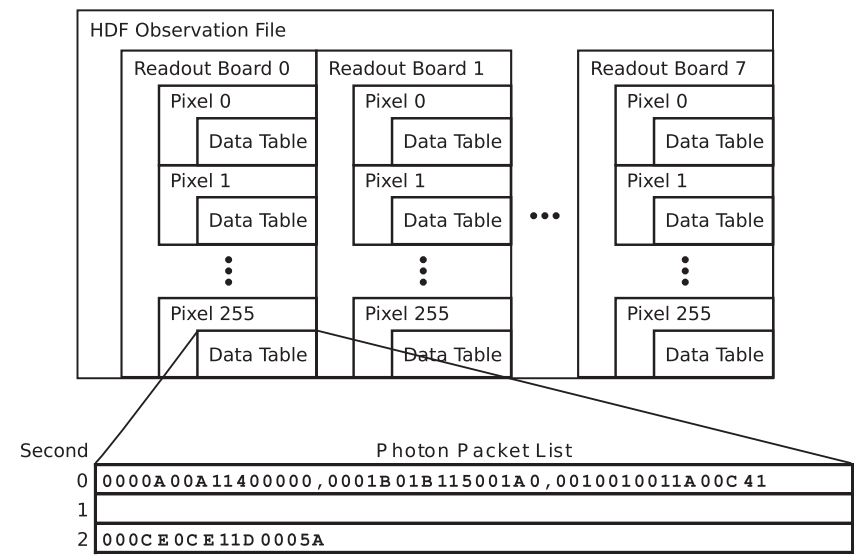

Figure 2. Organization of the raw data file created during observations. The file is organized first by readout board, then by pixel. Each pixel is allocated a data table, in which each row corresponds to a second of observation time. Each row in the data table has a photon data packet for every photon detected for the pixel during the corresponding time, stored as a ragged array.

\begin{tabular}{|c|c|c|c|c|}
\hline \multicolumn{1}{c}{12 bits } & \multicolumn{1}{c}{12 bits } & 12 bits & 12 bits & bits \\
\hline Pixel & Peak Phase & Unused & Baseline & Timestamp \\
\hline
\end{tabular}

Figure 3. Structure of the 64 bit packet generated during a photon detection. The pixel number is only 8 bits because it represents an index into a list of pixels assigned to a readout board, each of which can read out 256 pixels. The least significant bit (LSB) is shown as the rightmost bit.

generally throw out data where we do not have all the boards reading out simultaneously, effectively adding a second of down time between observation files. This issue has been greatly improved, so that in our last observing run, instances of slow boards were very rare.

After applying corrections, timestamps can be corrected to solar system barycentric times with the TEMPO2 pulsar timing package (Hobbs et al. 2006) using a custom plug-in that allows TEMPO2 to treat photons individually. TEMPO2 takes parameter files indicating the location of the observation and the ephemeris of the object observed.

\subsection{Beam Mapping}

During the MKID design process, each pixel is assigned a unique resonant frequency by systematically varying the design of the capacitor for each pixel. The pixels are then assigned to positions on a rectangular grid according to an algorithm that randomizes their locations, maximizing the physical distance between pixels with similar resonant frequencies to avoid cross-talk. In theory, this creates a known mapping of pixel resonance-frequency to chip location. In practice, however, random non-uniformities in the superconducting critical temperature across the array shift the resonant frequencies in unpredictable ways. We must measure the actual resonant frequencies after fabrication and then empirically determine which frequencies correspond to which pixel location.

We first find the resonant frequencies of all the pixels by using a vector network analyzer to sweep probe signals through the chip across all frequencies in a few-GHz band, and tag the dips in signal transmission that signify a resonance. The resulting list of resonances is used to map readout frequencies to indexed pixel numbers. To determine each pixel's location, we systematically illuminate the pixels using an $X-Y$ translation stage holding a photomask patterned with two long, orthogonal 
slits, illuminated with a beam of light from the backside. We scan these strips of light across the array one at a time, then use the time stream of each resonator's response to precisely determine the $X-Y$ position when it was illuminated. The end result is a beammap file which assigns a pixel number to its corresponding resonant frequency and physical grid location. The beammap is loaded as a two-dimensional (2D) look-up table during data analysis to map each pixel to its proper location in the image.

The beam-mapped raw data provide the input for the data reduction pipeline, which eventually produces fully calibrated photon lists. The steps involved in this calibration are detailed below.

\section{PIPELINE COMPONENTS}

\subsection{Cosmic-ray Cleaning}

We use the term "cosmic ray" to describe events where photons arrive in different pixels nearly simultaneously. We attribute these events to radioactive decays and muons interacting with the MKIDs' silicon substrate. When a cosmic ray interacts with a MKID or the crystal substrate, it deposits much more energy than an optical photon. This creates a burst of phonons which are seen by many of the MKIDs in the array as false high energy photon events. We therefore see a broad "flash" across much (or all) of the array, in contrast to the cosmic ray tracks commonly seen in conventional CCD images. These rare events are eliminated using the coincident arrival times with a loss of less than $0.1 \%$ of the total exposure time. The algorithm calculates time intervals within a data file that are near cosmic ray events and then excludes data in those time intervals from further analysis. The following parameters control the algorithm:

stride the number of consecutive output sample time steps combined to form one "time bin";

threshold the minimum number of photon packets in a time bin to determine whether a cosmic event is detected;

width the number of time bins to exclude around a detected cosmic ray event.

We read in the time for all of the photon packets from all of the pixels in a data file. We form time bins from consecutive time samples, starting at the first time step. We then form overlapping bins by starting at time step stride/2. For all of the time bins that have more than threshold photons, we calculate a time interval to exclude. The number of apparent photon detections as a function of time increases quickly in these events, with a longer decay tail. We exclude width time bins before the cosmic ray event and twice that length after the end of the time bin. We form the union of all of the excluded intervals of a data file and use this list of intervals to mask cosmic rays.

Figure 4 shows the distribution of the number of photons per time step for a typical data file. The solid line is for all photon packets, with no masking. Note that the population is dominated by a Poisson distribution, with $\mu \sim 0.5$. Cosmic ray events are in the tail of the distribution with population $>10$ photons/time step. We mask using time intervals calculated with stride $=10(10 \mu$ s for our current $1 \mu$ s time step $)$, threshold $=15$ photon events, and width $=10$ time bins $(100 \mu \mathrm{s})$. This eliminates events in the tail and excludes $0.02 \%$ of time intervals.

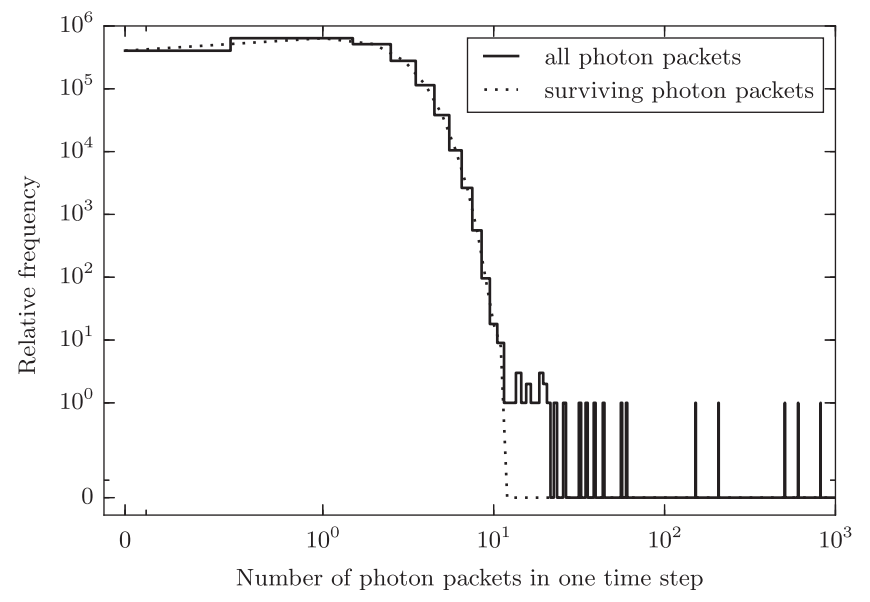

Figure 4. Distribution of number of photons per time sample. The solid line is all data (no cosmic ray masking) and the dotted line is after masking time steps near cosmic ray events.

\subsection{Bad Pixels and Exposure Time Masking}

It is found that some pixels in the array go "hot" from time to time, registering false events and reporting very high count rates, commonly over periods of order seconds (for the ARCONS 2012 data). We refer to these as "hot" pixels by analogy with the phenomenon of the same name encountered in conventional CCDs, albeit in the case of CCDs they tend to be more often permanent than transient features. In our case it is suspected that the effect is due to an interaction of the MKID with free electrons in the silicon wafer, which should be eliminated in future MKID designs.

In order to catch any such temporarily anomalous pixels, we use an algorithm similar to that employed in the "badpixfind" task of the XMM-Newton X-ray data pipeline. ${ }^{10}$ Because the point-spread function (PSF) of the telescope is oversampled by the instrument, any real astrophysical light source must present a minimum finite size on the detector. If the ratio of the flux in a pixel to the median flux in a surrounding box (say, $5 \times 5$ pixels) is significantly higher than could be expected for an astrophysical point source on the detector, then the apparent PSF is too narrow to represent anything real, and the pixel is flagged as hot and masked out. The threshold ratio (after accounting for sky background) is determined as the ratio of the peak of a model 2D Gaussian function to the median of the same function in a surrounding box of equivalent size. The FWHM of the Gaussian is set to match the expected seeing, and an additional ceiling is added to the threshold which accounts for both photon shot noise and the standard deviation of the measured sky background (typically at the $3 \sigma$ level). Using the standard deviation of the sky background helps to account for any internal instrument noise (due to variations in QE, etc.). Since neighboring bad pixels will skew the local median used for flux comparison, this process is iterated until there is no change in the mask or a maximum number of iterations is reached.

The flagging is performed in regular short integration timeslices (typically $1 \mathrm{~s}$ ) for each observation file, in order to capture the time variability of bad pixels and minimize the amount of good data that is unnecessarily flagged. The

\footnotetext{
${ }^{10}$ XMM-Newton Science Analysis System, Users Guide to the XMM-Newton Science Analysis System, Issue 9.0, 2012 (ESA: XMM-Newton SOC). See also http://xmm.esac.esa.int/sas/.
} 

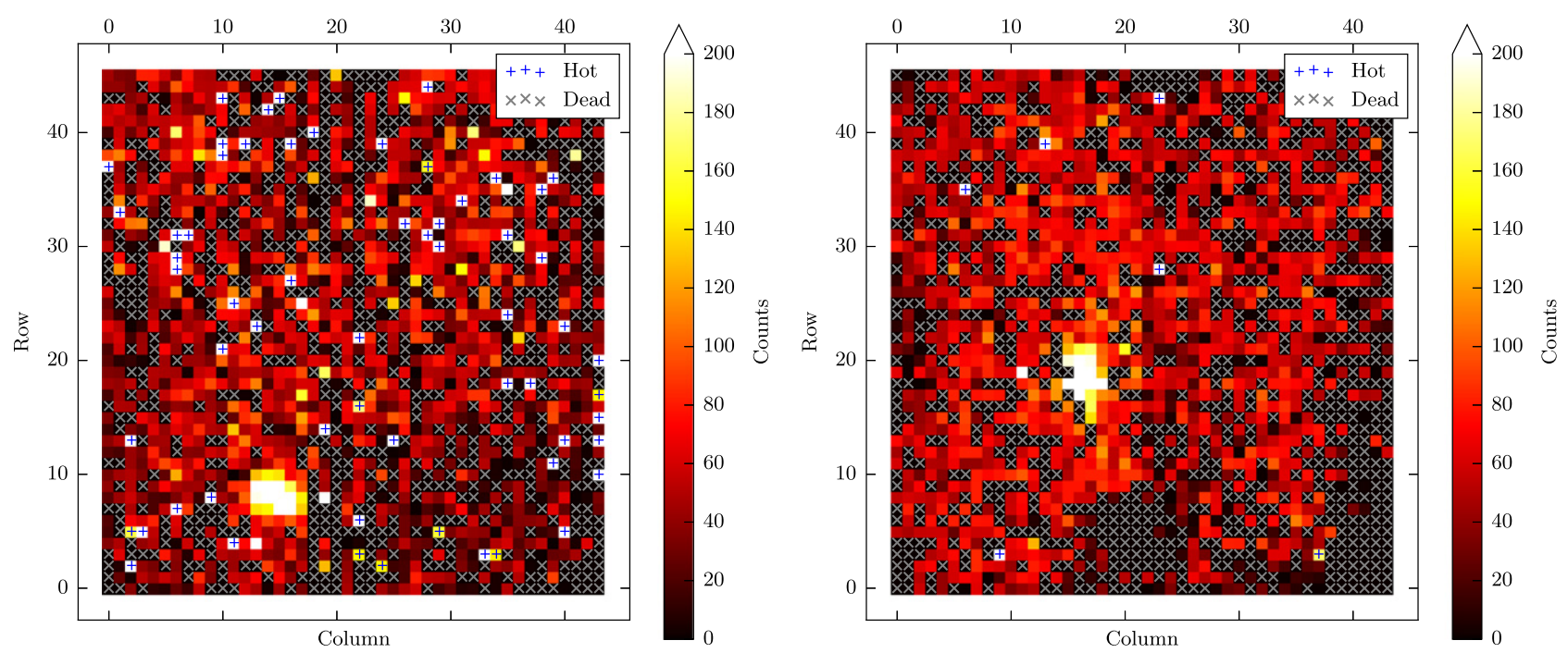

Figure 5. Bad pixels detected in example $1 \mathrm{~s}$ time-slices of raw data from ARCONS. Left: AM CVn binary SDSS J0926+3624, taken 2012 December 09 (UT). Right: compact binary SDSS J0303+0054, taken 2014 October 22 (UT). Pixels with high flux within the vicinity of astrophysical sources that are consistent with the PSF are not flagged, while others outside source vicinities are flagged as "hot." Improvements to the ARCONS array in 2014 led to substantially reduced hot-pixel behavior. Additional dead pixels in the 2014 data are are caused by magnetic flux trapping in the superconductors during array cool-down (the "hole" at bottom center), and by a fault in one of the eight readout circuit boards (bottom right of the array). Both these issues are correctable in future arrays.

resulting masks are recorded as a list of bad time-intervals for each pixel, creating a single time-mask file for each input raw data file.

"Dead" pixels - those recording no counts at all-are also flagged. In all cases, the reason for flagging is also associated with each recorded bad time-interval in the time-mask file. Current possible "reasons" are confined to dead pixels, hot pixels, and, optionally, "cold" pixels which may give abnormally low, but non-zero, count rates; there is facility to add further flagging as the pipeline matures. ${ }^{11}$

The algorithm is able to account somewhat for variability in $\mathrm{QE}$ from pixel to pixel, and the raw QE is reasonably uniform. However, it is better able to discriminate hot pixels with flatfielded data, where the noise levels are lower (see Section 4.4). Hot-pixel flagging can therefore be run either before or after the flatfielding has been performed (or both), depending on requirements.

The masks for two $1 \mathrm{~s}$ time slices of typical raw data files from 2012 and 2014 are shown in Figure 5, showing pixels that have been flagged as either hot or dead. For the same observation file from which the 2012 mask was derived, $\approx 8 \%$ of the pixels were flagged as exhibiting hot-pixel behavior at some point during the $300 \mathrm{~s}$ duration of the data file (of which $\approx 0.2 \%$ were flagged as hot for the entire $300 \mathrm{~s}$ ). The distribution of hot intervals began with a peak at $1 \mathrm{~s}$ (the sampling integration time) and tailed off toward longer durations. $31 \%$ of the pixels registered as "dead" for the duration of the exposure (including un-mapped pixels, etc.). Approximately 4\% showed temporary dead behavior, which seem largely to be the result of Poisson statistics for pixels which are registering extremely low count rates, either because they are faulty or because of exceptionally low quantum efficiency.

\footnotetext{
11 In certain cases, mislocated pixels resulting from beammap errors (Section 3.4) may cause a pixel from the core of the PSF of a star to be mismapped into a region of empty sky. In such a case, the pixel will also be flagged as "hot." Usually these mislocated pixels can be found and corrected in the beammap.
}

The new array used in the ARCONS 2014 run showed a significant improvement in hot pixel behavior. In the 2014 example image shown, only $\approx 0.5 \%$ of the pixels showed hot behavior at some point in the observation file $(0.15 \%$ for the entire duration). $41 \%$ were dead, an increase over the previous observations. This was due to magnetic flux trapping in the superconductor during cool-down and to a faulty readout circuit board, both of which can be corrected in future observing runs. Most of the remaining "dead" pixels in the arrays from both years result from collisions in the resonator frequency domain. This occurs because non-uniformities in the TiN film of the MKIDs shift the resonant frequency of pixels away from the design resonant frequency. When two or more resonators are less than $500 \mathrm{kHz}$ away from each other in resonant frequency we can only read out one of these resonators, leaving the others not addressed by the readout. More uniform superconducting films for MKIDs are currently being intensively investigated, and should drastically decrease the number of dead pixels.

The masking process does not capture all hot pixels: some may be hot but have flux levels which are marginally within the noise in a given time slice, and thus not identified. In principle, hot pixels have other characteristics which may be used in the future to further refine the flagging: the spurious counts oftenthough not always-have smaller event pulse heights, which the pipeline interprets as longer wavelength, so that spectral characteristics may help identify the behavior. Similarly, hot pixels often tend to switch "on" or "off" very suddenly (or change between various flux levels in a step-wise manner), which would be unusual for a true astrophysical source. Conceivably this timing and spectral information can be used to better identify bad pixels, particularly if it can be determined that neighboring pixels are not exhibiting the same behavior (since this would again tend to rule out an astrophysical source). It is also hoped that array development will mitigate many of these effects.

Future improvements to the detection of permanent bad pixels may be obtained by creating additional masks in a way 


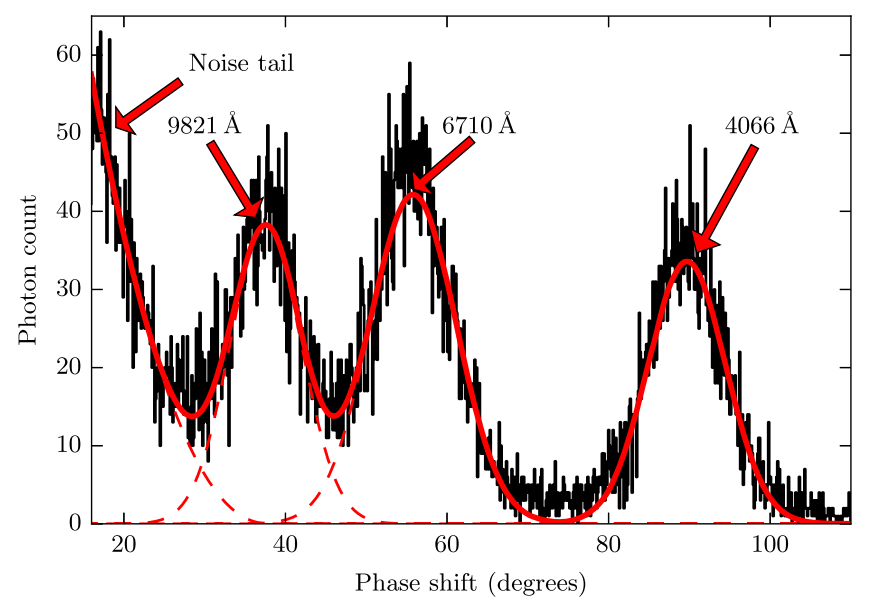

Figure 6. Histogram of a pixel's resonator phase shifts due to photon events from simultaneous exposure to a blue, red, and IR laser. Gaussians are fit to each laser's peak, and a parabolic fit to the locations of those peaks is used to convert phase shift to photon energy. A noise tail arises possibly from some combination of resonator baseline phase noise, an IR leak in the cold-filter transmission function, and photon interactions with the silicon substrate.

similar to traditional bad pixel masking for CCDs. By dividing two different flatfield calibration images at different flux levels (e.g., sky flats taken at different times during twilight), it is possible to identify outlying pixels whose flux response does not behave in proportion to the majority of well-behaved pixels.

\subsection{Wavelength Calibration}

Wavelength calibration involves determining the mapping from the photon-induced phase shift in a pixel's microwave probe tone to the photon's corresponding wavelength. For the purposes of calibrating the wavelength response for each pixel, 300 s wavelength calibration exposures are taken regularly throughout the night. These are created by uniformly illuminating the array with the combined light from a blue, a red, and an IR laser (currently at wavelengths 4066, 6710, and $9821 \AA$, respectively). For each pixel, a histogram of the phase shifts for every photon event in a given calibration exposure is accumulated, which shows three peaks corresponding to the three laser wavelengths (Figure 6). Gaussian functions are fitted to each of these peaks to find the locations of their respective maxima, which are taken to be the best estimate of the phase shift for the corresponding laser wavelength. A parabolic fit to these three phase values as a function of wavelength then gives a continuous estimate of the function mapping phase shift to wavelength for that pixel. The width of the Gaussian fits gives an estimate of the effective resolving power at each of the three wavelengths. A number of cuts and checks are performed to ensure a sensible fit, and any pixel which does not pass the checks is marked as failed. The results of the fits are stored as a calibration file, containing, for each pixel, the parabola coefficients, the wavelength range of validity of the calibration, any flags associated with the calibration (e.g., for pixels where the calibration fails), and a record of which $\mathrm{ROACH}$ readout board is associated with the pixel. A long wavelength cutoff is established at the point the count rate from the IR laser is equal to the noise, allowing noise counts to be ignored in later processing. Resolutions are typically $R \sim 6.5$ at $4000 \AA$, but range from $R=3$ to $R=10$ for different pixels.

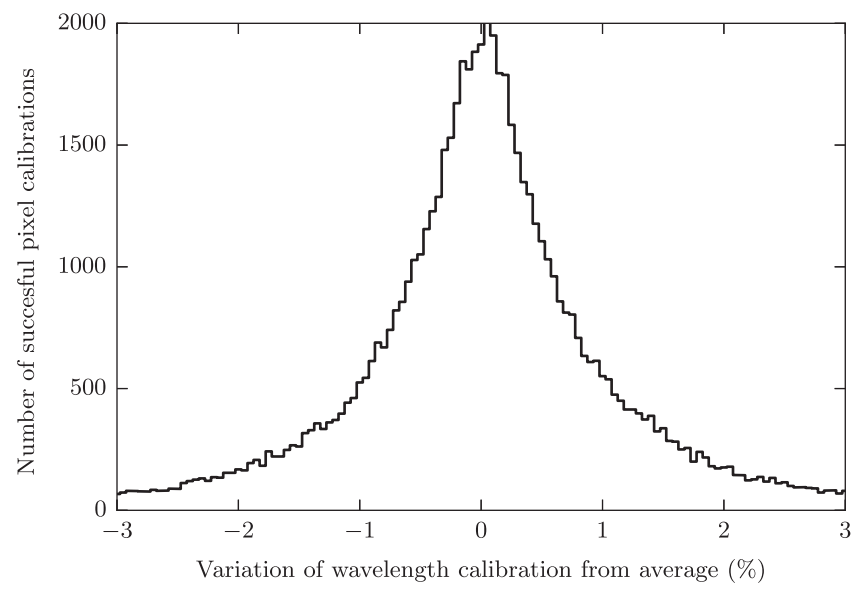

Figure 7. For each wavelength calibration exposure taken during a night, we find slightly different phase shifts caused by blue (4066 ̊) photons (See Figure 6). We determine the averaged master phase shift for each pixel and then calculate the variation for each single phase shift against the average. Here, we histogram this variation for every pixel and every wavelength calibration exposure to probe the overall array's wavelength calibration stability during the night. The distribution is centered around zero, indicating no apparent array systematics. The FWHM is about $0.5 \%$ which is small compared to the energy resolution of an individual photon of $\Delta E / E=15 \%$.

Properly accounting for photon pileup effects in the wavelength calibration data was found to be essential for a good solution. Failure to do so leads to significant broadening and skewing of the three histogram peaks, leading to systematic wavelength calibration errors and an apparent reduction in spectral resolution. This results from systematic errors in the measured phase baseline and phase shift (see Section 4.6.2). Such effects are corrected by removing photons that occur within a nominal $1 \mathrm{~ms}$ of the previous photon. The timescale of $1 \mathrm{~ms}$ can be shortened but it is more than long enough to remove any photon pileup effects.

Pixels with a valid wavelength solution have their Gaussian fit parameters saved in a calibration "drift" file. The stability of the wavelength calibration is determined from the pixel's response to the blue laser light over time. We find that throughout the night, most pixels' peak phase shifts for blue photons (as determined by the Gaussian fitting procedure) vary by less than $0.5 \%$ (Figure 7 ). If the hardware creating the pixel probe tones is restarted, peak phase responses can drift systematically by as much as $4 \%$. A detector array temperature change of about $5 \mathrm{mK}$ will systematically shift the peak phase response for all pixels by about $5 \%$. Since the temperature is controlled to better than $0.05 \mathrm{mK}$, temperature drift is not expected to be a significant source of error.

Since the pixel's wavelength calibration is quite stable over a night $(0.5 \%$ compared to $1 / R=15 \%)$, we average several wavelength calibrations into a single "master" wavelength calibration file. The UT times for which the master wavelength calibration file is valid are also saved. The wavelength of an observed photon is then determined by looking up the fit coefficients for the photon's pixel in the corresponding master wavelength calibration file, and using these to map phase shift to wavelength. The use of a master wavelength calibration has two main advantages. First, by averaging solutions from several wavelength calibrations we can get a more accurate calibration, assuming there are no significant systematic shifts in a pixel's phase response. Second, if a pixel has a bad wavelength solution for a specific wavelength calibration we 
can recover a solution by averaging the remaining successful wavelength calibrations.

\subsection{Flatfield Calibration}

The variations in response from one pixel to another are normalized using sky exposures during twilight before sunrise (we do not observe evening twilights to avoid accidentally heating up the detector array with too much light at the beginning of the night). Over the course of morning twilight, the sky's brightness and spectrum change dramatically, so the twilight data are broken into exposures of duration $20 \mathrm{~s} \mathrm{each}$, and data are only used until the average count rate rises above 800 counts per second per pixel. The brightness and spectrum of the sky are relatively stable during each of these exposures. Each of the $20 \mathrm{~s}$ data chunks is passed through the hot pixel module. Then, for each of the data chunks and each of the pixels with valid wavelength calibrations, the detected photons are binned into wavelength bins with widths equivalent to $0.1 \mathrm{eV}$. For each wavelength bin, the median count rate over all pixels is taken. This median is divided by the count rate in each wavelength bin in each pixel, yielding a set of weights, indexed by pixel and wavelength bin, for each of the $20 \mathrm{~s}$ data chunks. The weights of the different exposures are combined in a (weighted) $15 \%$-trimmed mean, to yield a final wavelengthdependent flatfield weighting for each pixel.

To apply the flatfield calibration to an observation, the observed number of counts for each pixel and wavelength bin is multiplied by its respective weight. This normalizes data from multiple pixels with respect to the average response, so that comparisons across pixels can be made validly. This affects the traditional CCD "flatfielding," but at every wavelength. When applied to observations of the sky, the most obvious effect of the flatfield calibration is to remove a gradient in pixel response thought to be caused by optical effects. This gradient is found in data from our 2012 observations but not in our 2014 observations, performed with a different detector array and re-aligned optics.

To characterize how well the flatfield calibration flattens the pixel response, we separate the large-scale effects of uneven illumination and intrinsic pixel-to-pixel variations by creating an alternative set of weights that corrects uneven illumination, but not pixel-to-pixel differences. To do this, we first smooth each twilight wavelength slice image with a sigma-clipped mean, and then proceed, as in our normal flatfielding, to take the median of the image and divide it by the image to generate the needed weights. The smoothed version of the wavelength slice image captures the large scale spatial variations in illumination but does not capture any pixel-to-pixel variation.

We can then characterize the two sets of weights by comparing the results of applying either of them to a 5 minutes night-sky exposure. We make images from the exposure showing the counts in the wavelength range 4000-11000 $\AA$ in each pixel without any weights applied, with the normal flatfield weights applied, and with the illumination weights applied. The distribution of counts for these images is shown in Figure 8. The distribution for the raw image does not have a sharp peak, because the large scale gradient smears it out. We compare the flatfield-calibrated and illumination-corrected distributions by taking the FWHM and converting it to a standard deviation, $\sigma$, with the assumption that the distributions are roughly Gaussian. We find that the illumination-corrected and fully flatfield-calibrated distributions have $\sigma$ values of

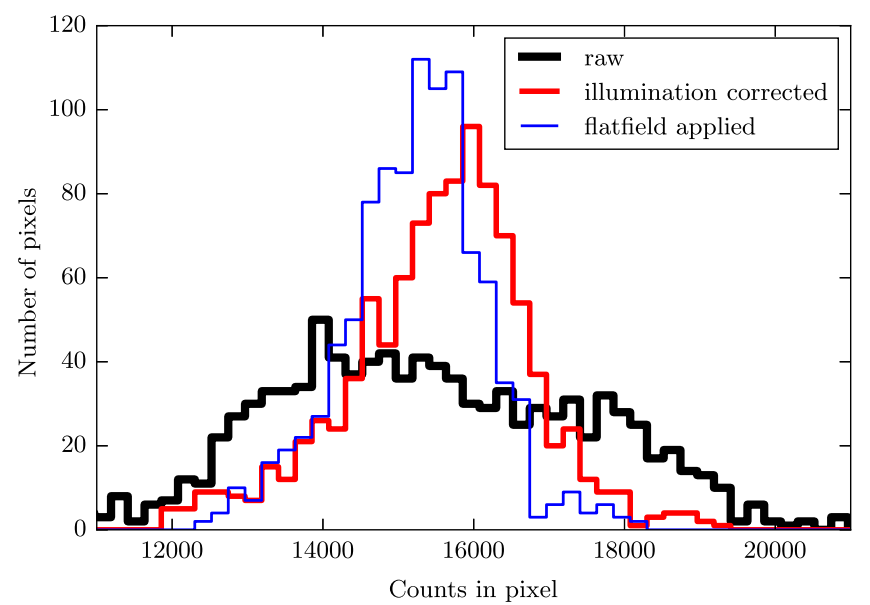

Figure 8. Distributions of pixel counts for images made from a 5 minutes sky exposure with no pixel response correction, illumination correction only, and with flatfield calibration applied.

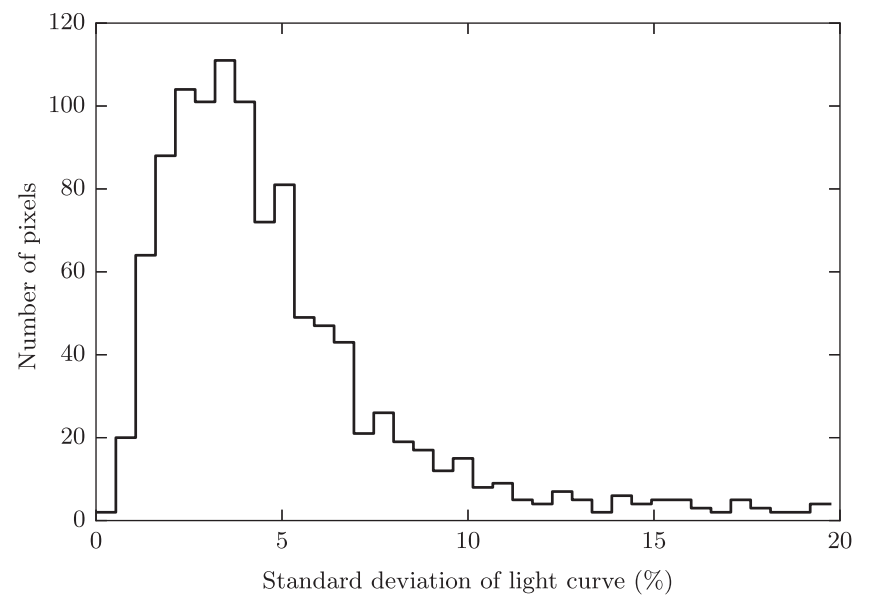

Figure 9. Distributions of standard deviations of pixel light curves of $2.5 \mathrm{hr}$ of night-sky exposures. The standard deviation expected from photon shot noise would be less than $1 \%$ for all pixels.

$6.1 \%$ and $5.0 \%$ of the median count rate, respectively. These values are relatively close given the uncertainty involved in computing them. For the median count rate in a pixel, we expect photon shot noise to contribute to the width with $\sigma=0.8 \%$. If the flatfield calibration were to remove all pixelto-pixel variation, we would expect the count distribution to have this standard deviation. Instead the flatfield calibrated distribution has a standard deviation that is comparable to the illumination corrected distribution. So, the flatfield calibration currently accounts for illumination correction, but does not significantly improve pixel-to-pixel uniformity.

To explain this, we checked the stability of a pixel's count rate with time. For this test, we used $2.5 \mathrm{hr}$ of night-sky exposures, divided into segments of $150 \mathrm{~s}$ each. A light-curve is made for each pixel, which is normalized by a reference light-curve made of the median counts over all pixels for each time segment. The standard deviation of the normalized light curve is then taken for each pixel. The distribution of standard deviations for all pixels is shown in Figure 9. For all pixels, the standard deviation expected from photon shot noise is less than $1.0 \%$. Nearly all pixels have standard deviations above this, indicating that there is some instability in the flatfield with time. The peak is close to the standard deviation of the flatfield- 


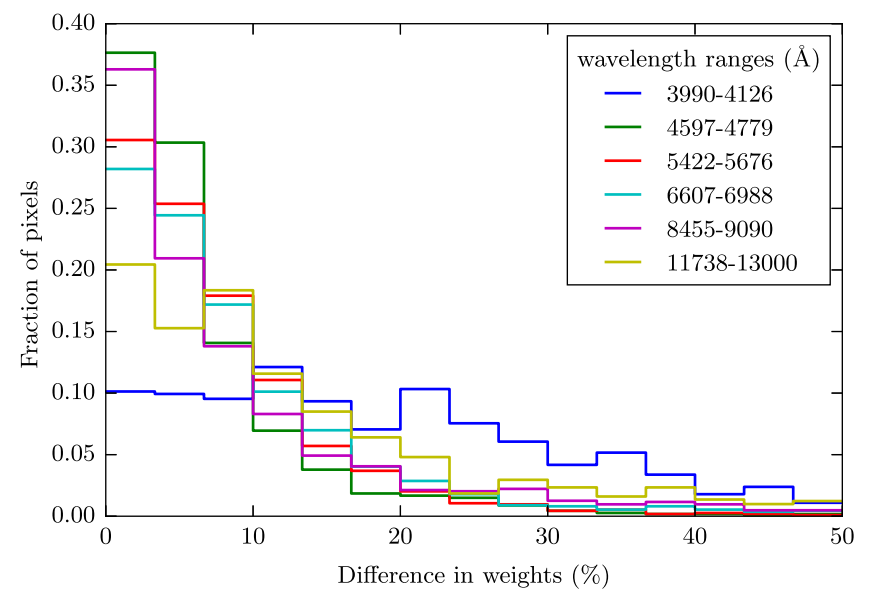

Figure 10. Distributions of percent differences between flatfield weights from one night's twilight and the next for a selection of wavelength bins.

calibrated count distribution from Figure 8, which accounts for the limited ability of the flatfield calibration to completely flatten pixel response.

We also investigated the stability of a pixel's calibrated flatfield weight from one night to the next. Figure 10 shows the distributions of percent differences between flatfield weights calculated for one night's twilight and for the next night. There is a sizeable fraction of pixels whose calculated weights change by $10 \%$ or more, particularly in the bins with the shortest and longest wavelengths. This is possibly due to changes in those pixels' wavelength calibrations from one night to another.

In a few pixels, the flux observed during twilight is not consistent with the QE observed indirectly at other times of the night, mostly likely due to those pixels having unreliable wavelength calibrations. These pixels receive flatfield weights either much lower or much higher than they should be assigned. The effect is that after the flatfield calibration is applied, these pixels appear hotter or colder than they should be. Because of this, and in light of the fact that in its current state the flatfield calibration does not generally improve pixelto-pixel uniformity, we currently only apply the illumination correction. The weights calculated for this correction do not have extreme changes from one pixel to a neighboring pixel, and in general, these weights also change less from one night to the next than weights calculated without smoothing.

\subsection{Spectral Response Calibration}

Once the pixel responses are normalized relative to one another by the flatfield calibration, absolute spectral shape is calibrated by dividing an ARCONS measured spectrum of a spectrophotometric standard star by the known spectrum of said target.

To extract the raw ARCONS spectrum we start with the standard processing, masking hot pixels and applying the linearity correction discussed in Section 4.6. Then the observation is broken into an image cube with wavelength dependent flat-field applied and spectral bands spaced $0.1 \mathrm{eV}$ apart. This band size matches the flat calibration binning, and over-samples the median MKID energy resolution by roughly a factor of 4 . We then PSF fit the stellar image in each spectral band and integrate the 2D Gaussian fit to determine the total flux from the object in that band.

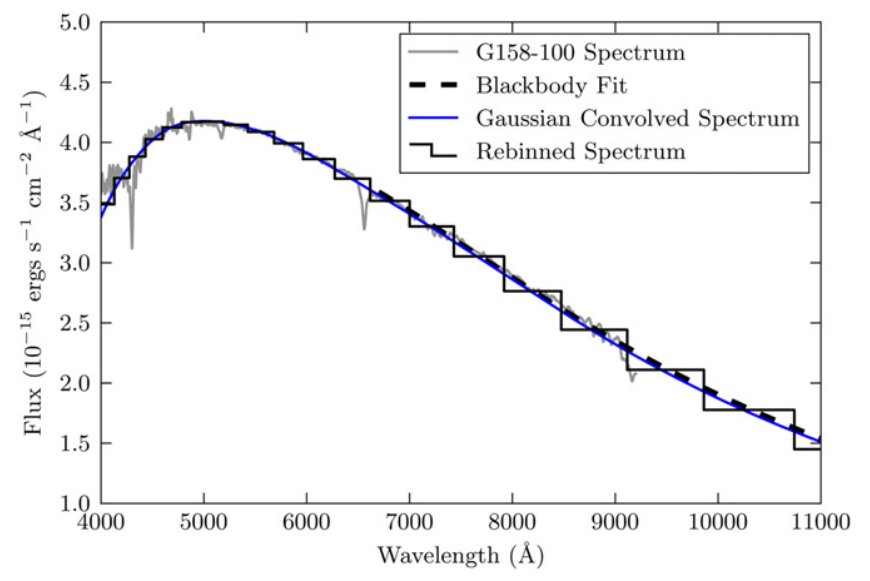

Figure 11. To prepare the known spectrum of a spectrophotometric standard (solid gray), it is first extended to fill our wavelength coverage using a blackbody fit (dashed black), then convolved with a Gaussian kernel with width determined by ARCONS' median energy resolution, corresponding to a width of $0.4 \mathrm{eV}$ (blue line). This low-resolution spectrum is then resampled by integrating over $0.1 \mathrm{eV}$ sub-bands, matching ARCONS flat calibration binning, and dividing by bin width in Angstroms to maintain proper $F_{\lambda}$ units (black steps). G158-100 archival spectrum from www.eso.org.

To prepare the known standard spectrum for comparison we first extend the near-IR coverage using a blackbody fit, since ARCONS' broad bandwidth extends to longer wavelengths than most commonly available spectrophotometric standard data. The pipeline can also accommodate model spectra covering any desired wavelength range, obviating this step. We then convolve the high resolution spectrum with a Gaussian kernel of width determined by the median energy resolution of the MKIDs ( $0.4 \mathrm{eV}$ width for 2012 data) to simulate how the spectrum would appear at ARCONS' median energy resolution, if QE was $100 \%$ at all wavelengths. This low-resolution spectrum is then resampled by integrating over sub-bands matching the flat calibration binning and dividing by bin width in Angstroms to maintain proper $F_{\lambda}$ units. Figure 11 demonstrates this process on G158-100, a dG-K type standard with $V=14.89$.

Dividing the two spectra yields a single whole-array weighting function designed to flatten the spectral response of the detector. When combined with the per-pixel relative flatfielding weights this spectral calibration also gives the overall QE of each pixel as a function of wavelength. Our 2012 spectral calibration curve is shown in Figure 12.

Applied together, the flat weights and spectral response weights yield an absolute spectrum for each pixel. Figure 13 demonstrates the generation of calibrated spectra on two wellcharacterized targets, Landolt 95-42 and CoRoT-18, with known photometry overplotted for comparison (Landolt 1992; Hébrard et al. 2011). For both targets, flatfielded data cubes were generated following the same procedure as the calibration star. The spectral response calibration was then applied as a single whole-array weighting per wavelength bin, and PSF fitting was performed on these fully calibrated spectral frames. This method of spectrum generation is suitable for observations with short integrations, such as the ones taken on these targets, where missing pixels are not filled in by dithering. The general approach for spectrum extraction from fully processed photon lists is described in Section 5.3.

As mentioned above, the spectral response calibration also serves as an integrated throughput measurement including the 


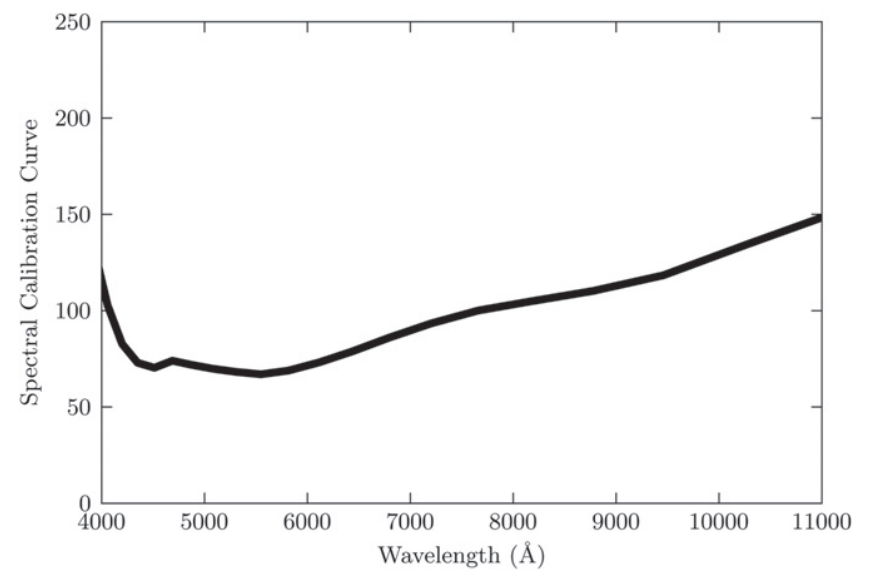

Figure 12. Spectral calibration curve from our 2012 run, generated by dividing the known spectrum of a standard star by the ARCONS measured spectrum (the inverse of the full system throughput). Flux calibrated spectra are generated by multiplying flat-calibrated spectra by this correction.

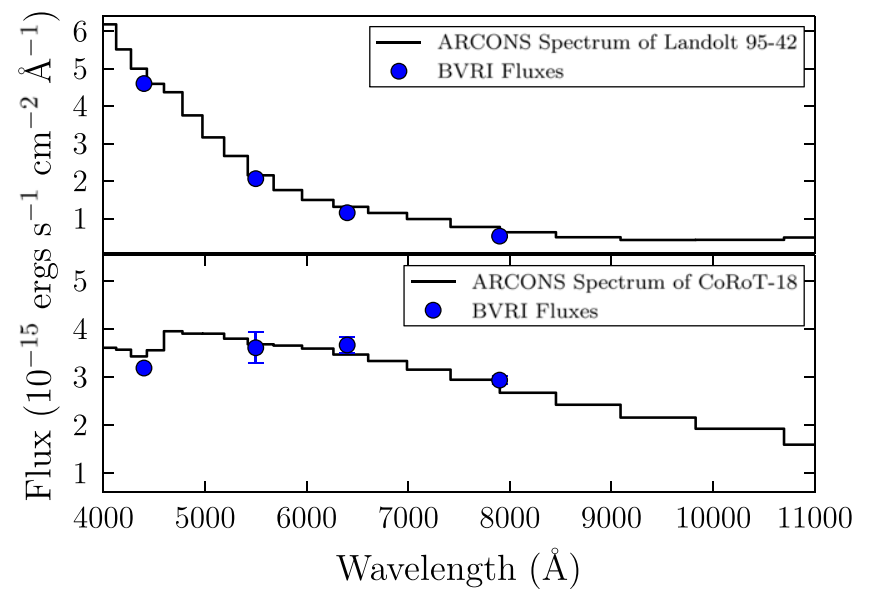

Figure 13. Flatfield- and spectral-response-calibrated spectra of known targets, Landolt 95-42 (Landolt 1992) and CoRoT-18 (Hébrard et al. 2011), demonstrating the accuracy of the spectral shape correction. It is worth noting that the cited CoRoT-18 B-band magnitude has no error bars provided.

atmosphere, telescope, camera optics, and detector. During our 2012 run, we found the on-sky throughput, as measured during a 5 minutes observation of the spectrophotometric standard star G158-100, to be roughly a factor of 2 lower than expected (Mazin et al. 2013). When we returned in 2013 we performed a more careful accounting of our light loss as shown in Figure 14. We measured the telescope throughput independently through Johnson B, V, and R filters using a UDT Instruments Model 221 Silicon Sensor placed just before the ARCONS entrance window, read out with a UDT Model S480 flexOptometer. The measured throughput values of $20 \%-30 \%$ are roughly 2 times lower than we expected, matching previous studies of aluminum coating degradation over many years (Magrath 1997) and accounting for our extra light loss. We have attributed most of this degradation to the Coudé flat mirror, which has since been re-aluminized and should now be restored to a pristine state. Due to poor atmospheric conditions on the 2013 run, and the only available spectrophotometric standard star, HZ21, being too faint at red wavelengths, we were not able to achieve an on-sky throughput measurement across our entire bandwidth

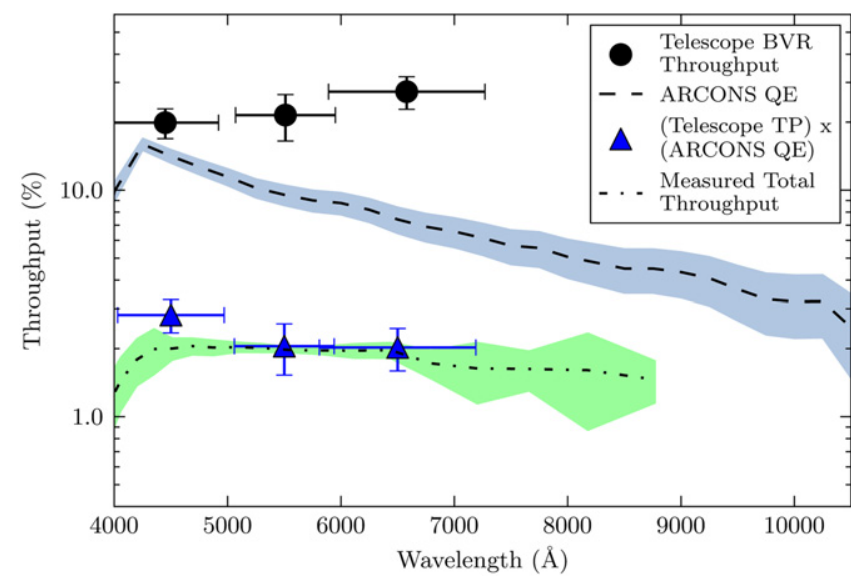

Figure 14. Full accounting of light loss performed with data from our 2013 run. Independent measurements of the Palomar 200" Coude B, V, and R throughput (black points) are shown with max/min error bars from a few data points. ARCONS lab QE (MKID + camera optics, dashed line) is shown with estimated $+/-1 \%$ error due to potential alignment error of the QE testbed. Telescope throughput multiplied by ARCONS QE gives a calculated total throughput estimate (blue triangles). This estimate shows good agreement with the measured on-sky throughput (dashed-dotted). (For more detail on MKID and camera-optics QE, see Mazin et al. 2012, 2013.)

even with a factor of 4 times longer integration than in 2012. However, the data shown here still demonstrate that the combination of independent telescope throughput and ARCONS QE (optics + detector) measurements matches the final on-sky throughput. Together, these data give a complete picture of where light is lost during our observations.

The QE of the MKIDs is simply the fraction of photons that are absorbed in the TiN metal. Since MKIDs are fundamentally sensitive to energy and do not need to carefully keep track of a photoelectron like a CCD, it should be possible to use absorbers to increase the $\mathrm{QE}$ significantly. We are currently investigating vertically aligned carbon nanotube absorbers (VACNT; Yang et al. 2008) that promise nearly 100\% QE across the entire UVOIR wavelength range.

\subsection{High Count-rate Correction}

Since MKIDs are single-photon counting devices they can encounter problems at high count-rates including photon pileup, data transfer rates, and pulse triggering. With ARCONS' 2024 pixel array and $1 \mu$ s sample rate, it must apply photon pulse triggering in the firmware for manageable data storage after a full night of observations. Individual pixels are limited to count rates of $2500 \mathrm{cps}$ to avoid any data transfer backlogs. We find pixels will saturate on $m_{V}=14$ stars with a throughput like Figure 14 and nominal 1".5 seeing. Brighter stars are observed by defocusing the telescope or using a neutral density filter. Our firmware is made to trigger on single photon events and can be nondeterministic under conditions of photon pile-up. We employ a dead time to avoid triggering on a single photon twice, and to cut out photons riding on the tail of the previous photon's phase pulse. This simple event detection algorithm and subsequent dead time causes a nonlinearity in the detector's intensity response, and can also systematically lower the apparent height of a photon's phase pulse. 


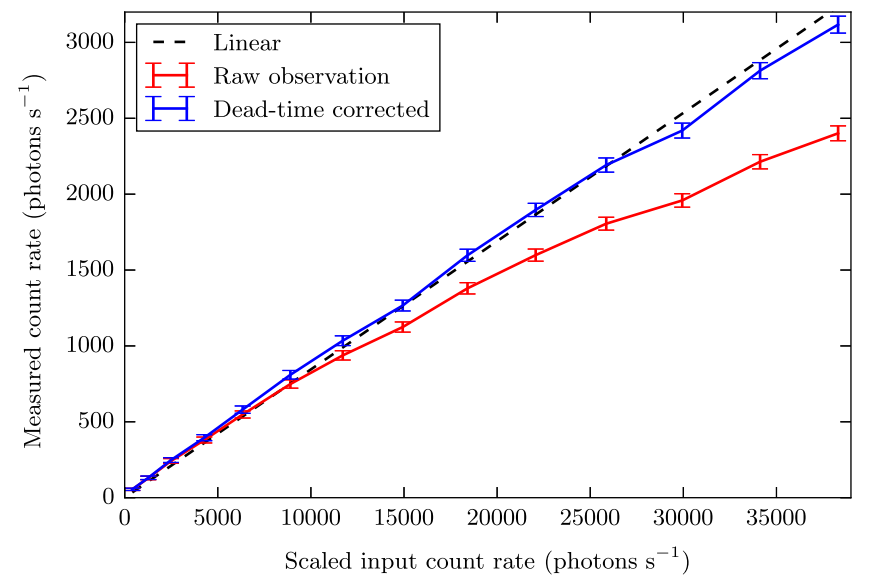

Figure 15. ARCONS flux response linearity. Scaled input count rate is the flux per pixel incident on ARCONS from a monochromator at $5500 \AA$, as measured by a calibrated photodiode. Measured count rate is the average number of photons recorded by ARCONS per pixel. The red connected points show raw data while the blue is dead-time corrected. The dashed black line shows the expected count rate for a perfectly linear detector with $8.5 \%$ quantum efficiency. This figure is reproduced with data from Mazin et al. (2013).

\subsubsection{Detector Nonlinearity Correction}

Photons arriving within a given time (usually 10-100 $\mu \mathrm{s}$ ) of a preceding photon on the same pixel are locked out by design in the firmware, creating a non-paralyzing ${ }^{12}$ dead time (see Section 3.1). At high count rates, this systematically lowers the effective quantum efficiency. A chunk of time, either within an exposure time or spanning multiple exposures, can be corrected by weighting count rates by a factor of $(1-N \cdot \tau / T)^{-1}$, where $N$ is the number of detected photons in exposure time $T$ for a pixel with dead time $\tau$. Figure 15 shows the linearity response before and after dead time correction. Both the flatfield calibration and spectral response calibration employ a $100 \mu$ s dead time correction (10 $\mu$ s for more recent 2014 data).

\subsubsection{Wavelength Bias Correction}

The baseline of the phase signal from a pixel, recorded with a low-pass filter in the firmware (see Section 3.1), is systematically changed by the phase shift of an arriving photon. This has the effect of raising the phase trigger threshold, and hence the minimum trigger photon energy, for a photon pulse arriving within several hundred microseconds afterwards. This timescale is set by the timescale of the lowpass filter. The baseline shift is small enough, and the trigger threshold is set low enough, that we do not lock out photons within our wavelength band. However, photons arriving within several hundred microseconds of a previous photon event appear to yield smaller phase shifts due to an artificially raised phase baseline, and are thus systematically biased redward after wavelength calibration. A longer low-pass filter timescale can make the effects smaller but the timescale must be short enough to follow real shifts in the baseline which occur on millisecond timescales. For most objects, with count rates less than $1000 \mathrm{cps}$, this effect can be ignored. For high count rates, the baseline is biased further with increasing count rates, as

\footnotetext{
${ }^{12}$ Photons arriving during that interval do not reset the lock-out; if they were to do so, then very high count rates would prevent any photon triggers, and the detector would be "paralyzed." A non-paralyzing dead time instead continues to allow event triggers, but at a rate limited by the dead time.
}

increasingly coinciding photon events skew the average phase signal. Such effects can be corrected by using only photons which have no other photon events occurring within a nominal period of $1 \mathrm{~ms}$ prior to their detection (provided the count rate is not so high that such photons never occur).

\subsection{Astrometry}

Flexure and hose-routing constraints imposed by the ARCONS cryostat mean that ARCONS must be located at the telescope Coudé focus at Palomar, and so the field of view (FOV) rotates continuously with time. Guiding is generally performed in such a way as to keep the target object in a fixed position on the array, and the rest of the field rotates around this position in step with the hour angle of the observation. In order to assign R.A. and decl. coordinates for each photon detection so that long integrations can be properly stacked, it is necessary to establish an astrometric solution. This can be approached in two ways, using either the main ARCONS detector images or using images from the guide camera, depending on the brightness of sources available in the ARCONS FOV.

\subsubsection{Science Camera Astrometry}

Where a bright source is present that can easily be detected in short integration times, a pointing origin is first established with a precise determination of the object centroid pixel location on the ARCONS array. To find the centroid position, we use the Python package PyGuide. ${ }^{13}$ PyGuide works by finding the point on a 2D image that minimizes the radial asymmetry of the flux, while accounting for missing data (primarily caused in ARCONS by unassigned and badly behaved pixels). The centroid location, in pixels, of the target object is associated with the object's known catalog R.A. and decl. The centroiding precision is $\lesssim 1$ pixel $\left(\lesssim 0^{\prime \prime} .5\right)$ for a $g^{\prime}=19$ magnitude object at $1^{\prime \prime} .5$ seeing.

The orientation of the FOV comes directly from the object's hour angle at the time of observation (with a possible constant offset depending on the exact physical orientation of the detector at installation). The FOV orientation, the origin obtained from the centroiding, and the detector plate scale $(\approx 0 \prime$. 44 per pixel for ARCONS at Palomar, from measurements of the pixel separation of known visual binaries) together completely determine the astrometric solution. A list of photonevent pixel coordinates can then be transformed into R.A. and decl. coordinates using linear matrix translation and rotation operations.

This procedure assumes that there is negligible field distortion on the scale of the science camera FOV. In fact there is known to be a small amount of distortion across the field, perhaps up to $\sim 0^{\prime \prime} .5$, along with a certain amount of chromatic aberration; this remains to be fully characterized, however. In principle calibrations of the larger guide-camera FOV, discussed in the next section, could be used to remove this effect.

The images used for the astrometric solution are created by integrating over a specified duration, chosen to be short enough that field rotation is small ( $<1$ pixel). This sets the sampling rate of the astrometric solution time series. The default astrometric solution time is 30 seconds. Faster times on

\footnotetext{
${ }^{13}$ http://www.astro.washington.edu/users/rowen/PyGuide/Manual.html
} 
brighter objects may allow effective software tip/tilt corrections to improve the recovered object PSF.

\subsubsection{Guide Camera Astrometry}

In order to find objects that are too faint to see on the science camera in short exposures (i.e., shorter than the field rotation timescale), we can use guide camera images to calculate the expected position of the object on the ARCONS science array, since the location of the ARCONS array is fixed with respect to the guide camera detector. The guide camera used in ARCONS is a SBIG STF-8300M CCD camera with a $1 ! 5$ FOV. Pick off mirrors positioned to either side of the ARCONS dewar entrance fold an image back to the guide camera, which sees a wider field than ARCONS. An empty strip down the center of the guider image marks the gap between the mirrors, within which the ARCONS detector itself lies. Each guide image is saved as a standard FITS file. Exposures of up to $10 \mathrm{~s}$ are used to reveal stars down to 20 th magnitude. Knowing the catalog R.A./decl. of the observed guide objects along with parameters relating guide camera positions to ARCONS positions, it is possible to assign R.A./decl. coordinates to individual ARCONS pixels.

In practice, it is first necessary to measure and eliminate all field distortions, which become significant on the scale of the guide camera FOV. We use the Simple Imaging Polynomial image distortion convention (SIP; see Shupe et al. 2005), which uses a set of polynomials to characterize the distortion. We use Source Extractor (Bertin \& Arnouts 1996) to automatically locate objects in the guide images; if the guider field is crowded or the objects are highly distorted, the code also allows for manual extraction of objects within the field. In both cases, we query the online VizieR service ${ }^{14}$ for object coordinates (currently primarily using the 2MASS and USNO-B1.0 catalogs, depending on source brightness). The objects in the catalog then need to be matched with those found in the guider field. To facilitate automatic processing, a list of potential match patterns between the catalog stars and guider field stars is constructed. For each potential match pattern, both a linear offset and a rotation are applied to minimize the distance between catalog and image stars, and the corresponding total of the squared residuals is recorded. The pattern with least total error is used to associate objects found in the guider field with those in the catalog. Manual matching is also an option if the process fails.

In the FITS convention, any distortion parameters should not contain zeroth or first order correction to an image. These two corrections are included in the standard header keywords $C D$ and CRVAL (which describe offset, rotation, and linear scaling). Linear offset and rotation are therefore first applied to each guider image to eliminate lower order corrections. The distortion polynomial is then calculated using multiple fields observed at various times throughout an observing run, again by minimizing the sum of the squared distances between matching stars in the catalog and the guider field. Unlike offset and rotation, the distortion parameters are expected to hold approximately constant during a run, as the optics setup remains unaltered during observations. (Since ARCONS is currently removed from the telescope between runs, alignment of the optical train is not, however, expected to be consistent from run to run.)

\footnotetext{
${ }^{14}$ http://vizier.u-strasbg.fr/viz-bin/VizieR
}

To construct a coordinate grid on the ARCONS array, we need to know the location of at least one guide image pixel in the ARCONS pixel coordinate frame. Since the ARCONS array itself cannot be seen in the guider images, we manually determine the position of a known bright star on the ARCONS array and compare it to the expected location in the guide images, and from that obtain a reference point. Once this fixed reference point is calibrated, and assuming the respective plate scales of ARCONS and the guide camera are known, it is then possible to assign R.A./decl. coordinates to each ARCONS pixel, and extrapolate the position of faint objects in the science array. In principle, knowledge of the field distortion established from the guide camera can also be used to mitigate the effects of distortion in the science camera astrometry; this has yet to be implemented, however.

At this point the technique of obtaining astrometric solutions for the ARCONS science array using guide camera images remains somewhat experimental, and has not yet been fully integrated into the standard pipeline; currently, astrometry for long exposures and mosaics (Section 5.1) is registered using bright sources in the science camera images.

\subsection{Calibrated Photon List Creation}

Once all calibrations have been applied, the data are written out as fully calibrated photon lists, one HDF5 file per input raw file. Each list is stored as a single large table, with one row per photon, and columns recording various data about each photon, including: photon arrival time relative to the start of the data file; original $x, y$ pixel location on the detector; R.A. and decl.; hour angle (used later for image reconstruction purposes); wavelength; weights for the photon associated with flatfield and spectral response calibrations; and any flags from the photon calibration process. Unusable photons, such as those falling within hot or bad intervals identified in the exposure time masks (Section 4.2), or pixels with failed wavelength calibrations, are omitted from the list in order to conserve data storage space. (The storage space required increases in proportion to the total flux recorded, so hot pixels in particular can have a significant impact on total storage requirements.) Stored alongside the photon list table in each photon-list file is a copy of each of the calibration files used in creating the list, which can be used to trace back and debug a given reduction. The relative size of these calibration files is small enough that the resulting duplication of calibration information across different photon list files does not have a major impact.

\section{APPLICATIONS OF CALIBRATED PHOTON LISTS}

The calibrated photon lists described in the previous section constitute the basic data product from which science data products can be derived. In this section, we show the use of these photon lists to produce examples of imaging, time-series photometry, and spectroscopy that show the multi-dimensional capabilities of MKIDs. In particular we show results from commissioning observations of the Crab Pulsar, a target which provides for all three applications using the same dataset. We also show light curves of the eclipsing AM CVn binary SDSS J0926+3624 from the same commissioning run. These demonstrate multi-bandtime series photometry on timescales of order hours, in comparison to the fast $33 \mathrm{~ms}$ period of the Crab Pulsar. 


\subsection{Imaging}

\subsubsection{Image stacking}

The photon arrival time resolving capabilities of MKIDs can simplify observing in many ways: the telescope can be dithered, nodded, offset, or even repointed while the detector is exposing, since these can all be accounted for in software after the fact. Guiding errors are of little concern (unless it is required to maintain a image on the same pixel at all times), and field rotation is likewise easily handled. Given enough flux, even simple tip-tilt correction can in principle be applied $e x$ post facto to improve image quality, though this capability remains to be developed in the pipeline.

For long exposures and any kind of data stacking from separate observations, it is essential to perform derotation and pointing corrections. An approach somewhat analogous to the Variable-Pixel Linear Reconstruction ("Drizzle") algorithm of Fruchter \& Hook (2002) is used: flux from each detector pixel is remapped ("drizzled") onto a more finely sampled "virtual pixel grid" after rotating and rescaling, accounting for the overlap areas of the original and virtual pixels to conserve flux. In this case, however, instead of drizzling flux (a continuous quantity) onto the virtual grid, we are drizzling discrete photons.

Each photon in a calibrated photon list has an assigned R.A. and decl. corresponding to the center location on the sky at the time of detection for the detector pixel on which the photon was recorded. In the absence of further knowledge of the responsivity across the surface of the pixel, we assume that the detected photon could have landed anywhere within its footprint with equal probability. Knowledge of the hour angle at which the photon was observed allows us to establish the orientation of the pixel on the sky. We can therefore construct two unit vectors corresponding to the directions of the two axes of the pixel on the sky, and add a uniform random offset (positive or negative) along each of these unit vectors, so that the photon is positioned randomly within the footprint of the detector pixel in R.A. and decl. This is repeated for all photons. A simple 2D histogram of the resulting R.A., decl. coordinate pairs then results in an image remapped onto a virtual grid in sky coordinates, where the counts in each virtual pixel correspond to the number of photons that have landed in that pixel. The individual photon weights from flatfielding and spectral response rectification are accounted for by weighting the histogram, so that instead of each photon being considered a single count, it is multiplied by its respective weights. Flux is naturally conserved, and is automatically distributed across the virtual pixels in a way that accounts for their overlap area with the detector pixels. Although we are drizzling discrete photons, we note that applying the weights will still generally yield noninteger counts, just as for conventional CCD image flatfielding; attempts to round the data at any stage will only add artificial quantization noise.

Since the ARCONS detector oversamples the PSF of the telescope image, loss of image resolution as a result of the image reconstruction process, as discussed by Fruchter \& Hook (2002), is not a great concern. However, it is straightforward in principle to emulate the effective "shrinkage" of the detector pixels suggested by the same authors to further mitigate resolution loss, by simply reducing the range of the random offset added to each photon location. This has not yet been tested in the ARCONS pipeline.

\subsubsection{Exposure Time Weighting}

As the ARCONS FOV rotates and shifts, the detector footprint will rotate and shift on the virtual image plane: dead pixels and the edges of the detector will drift across the virtual image, so that some virtual pixels will see more total exposure time than others. To produce a useful image, therefore, it is also necessary to weight by the effective exposure time in each virtual pixel. To produce an exposure weight map for the virtual image, we slice the original exposure into short time steps exactly matching the time steps used for calculating the photon astrometry. Exposure time for each time step can then be thought of as a conserved quantity analogous to flux. The effective exposure time for each detector pixel within the time step is calculated based on the time masks described in Section 4.2, so that periods when a pixel went "bad" are also excluded. Detector pixels which are permanently bad are also automatically excluded in this way. These effective exposure times are then distributed across the virtual output image grid in proportion to the respective overlap areas of the detector pixels, in the same way as flux is in the more conventional "drizzle" scheme, where flux is handled as a continuous quantity (rather than the individual photon events described above). The detector/virtual pixel overlap areas are calculated using the Fortran routine "boxer," borrowed from the "PyDrizzle" package in the STSCI_PYTHON distribution. ${ }^{15}$

Multiplication of the virtual stacked image by the exposure weight map (or equivalently, division by the per-pixel effective exposure times) then gives an exposure weighted image which accounts fully for dead areas, missing data, etc. Figure 16 shows a full image stack from $\approx 3 \mathrm{hr}$ of integration time on the Crab Pulsar, split into three wavebands and rendered as an RGB image.

\subsection{Photometry}

The ARCONS pipeline provides photometry by both PSF fitting and aperture photometry. For observations where highcadence light curves are desired, PSF fitting or aperture photometry can be performed on data cubes generated directly from the observation files, still in detector coordinates. For observations with long total integration times, where field rotation is significant, or where dithering has been performed, we can perform standard aperture photometry on the fully calibrated photon-list generated image in R.A./decl. coordinates.

For most light curve analyses, we are interested in short effective integrations, meaning there is not sufficient time for field rotation or dithering to fill in missing pixels. In these instances we can use PSF fitting to estimate the missing flux. In its current state this module fits a circular 2D Gaussian to the desired wavelength slice in the data cube, still in detector coordinates. The baseline in this fit is subtracted off, removing the sky background, and then the amplitude and width of the fitted Gaussian are used to calculate the flux from the object.

When observing bright targets, such as planet transits, we must defocus the telescope to prevent count-rate saturation, often by a substantial amount. We are currently developing a robust module to perform PSF fitting on non-Gaussian PSFs to analyze such observations. This development is ongoing and is not included in the current pipeline release. An example series

\footnotetext{
${ }^{15}$ See http://www.stsci.edu/institute/software_hardware/pyraf/stsci_python.
} 


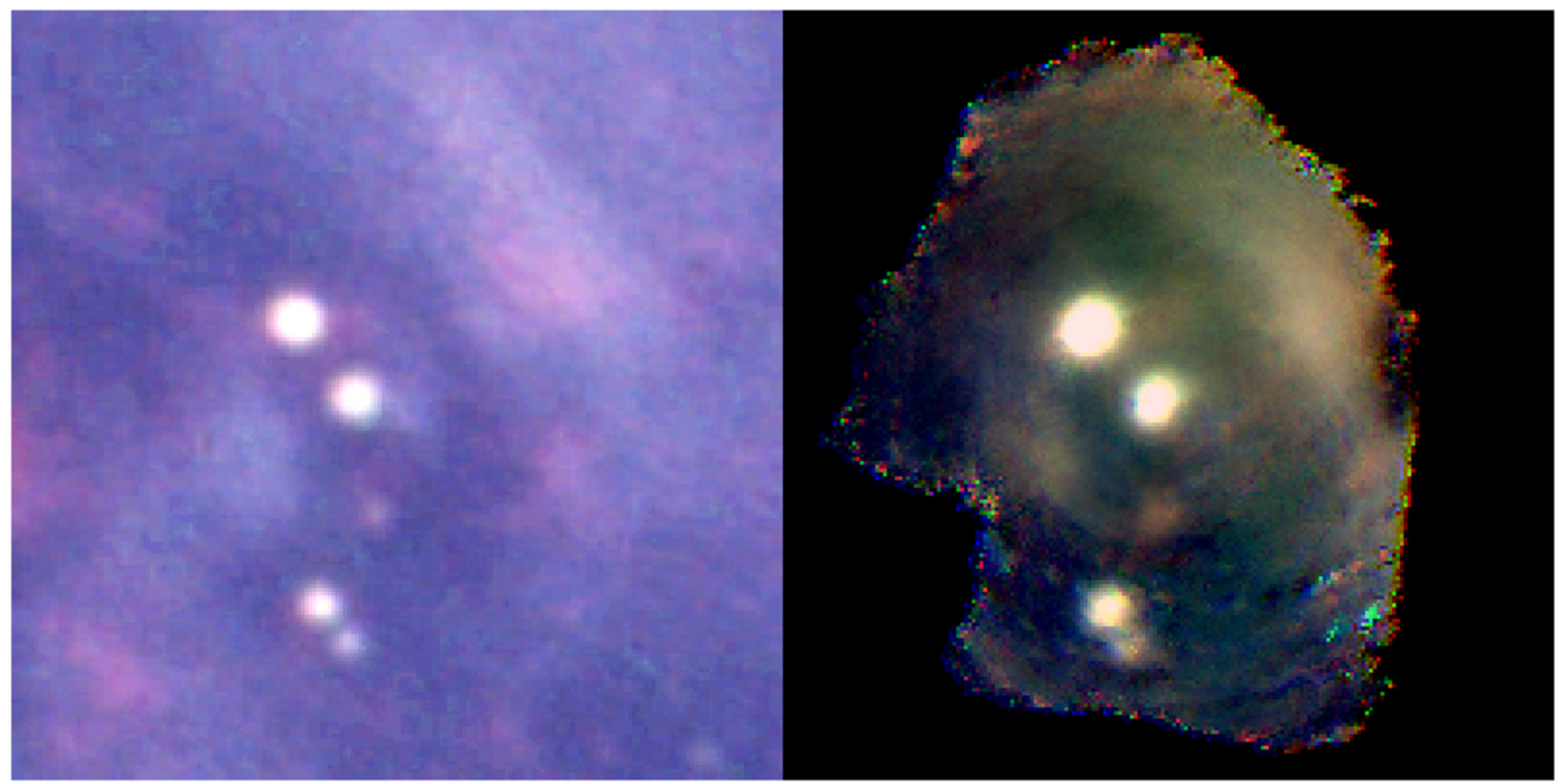

Figure 16. Left: image of the Crab region taken with Ektachrome color film at the prime focus of the Kitt Peak $4 \mathrm{~m}$ telescope (Credit: Bill Schoening/NOAO/AURA/ NSF, 1973). Right: stacking of $\approx 3 \mathrm{hr}$ of exposure time on the Crab Pulsar taken with ARCONS in 2012 December. RGB rendering is created from stacked photon lists in three wavebands: $4000-5000 \AA$ (blue), 5000-6000 $\mathrm{(green),} \mathrm{and} \mathrm{6000-7000} \AA$ (red). The data are split into 5 minutes chunks (matching the original observation files), which here are median combined to help minimize the effect of uncaught bad pixels. Streak-like artifacts in the image are due to remaining hot or otherwise badly behaved pixels that have not been handled by the pipeline and remain to be addressed. Higher noise around the image edges is caused by the low total effective exposure time in those areas. Since there is no atmospheric dispersion correction in the optics train, ARCONS resolves the differential refraction; the RGB component images have been reregistered by hand to reduce the effect, but some residual dispersion can still be seen.

of multiband light curves from the eclipsing AM CVn star, SDSS J0926+3624, created using our PSF fitting pipeline, is shown in Figure 17 (for more detail, see Szypryt et al. 2014).

For applications where PSF fitting fails (e.g., due to high sky background when analyzing far-red slices of our data cubes), we can apply standard aperture photometry to the data. This method is only applicable if the telescope pointing is stable enough to maintain the object's position on the same pixel over the course of the observation. Otherwise the PSF will drift over a changing pattern of missing pixels and PSF fitting is required to estimate the varying amount of missing flux. In our experience the Hale 200" telescope at Palomar is stable enough for us to maintain our target position to approximately one of ARCONS' $\approx 0$ ". 44 pixels.

For observations where short exposure times are not required (or not feasible), we can perform aperture photometry on the fully calibrated photon list images in R.A./decl. space where the astrometry for each photon has been fully determined. Figure 18 shows the broadband optical pulse profile of the Crab Pulsar derived from performing aperture photometry on the calibrated photon lists that produced Figure 16. In this case the timestamps of all photons in the aperture were folded on the pulsar period using an ephemeris derived from the simultaneous radio data (see Section 3.3), in order to build up sufficient signal. The dithering and field-rotation during the observations mean that missing pixels are not a concern. More details can be found in Strader et al. (2013).

\subsection{Spectroscopy}

Spectroscopy can be performed on a multi-dimensional image stack. During the photon stacking process, there is an option to include a wavelength dimension along with the R.A.

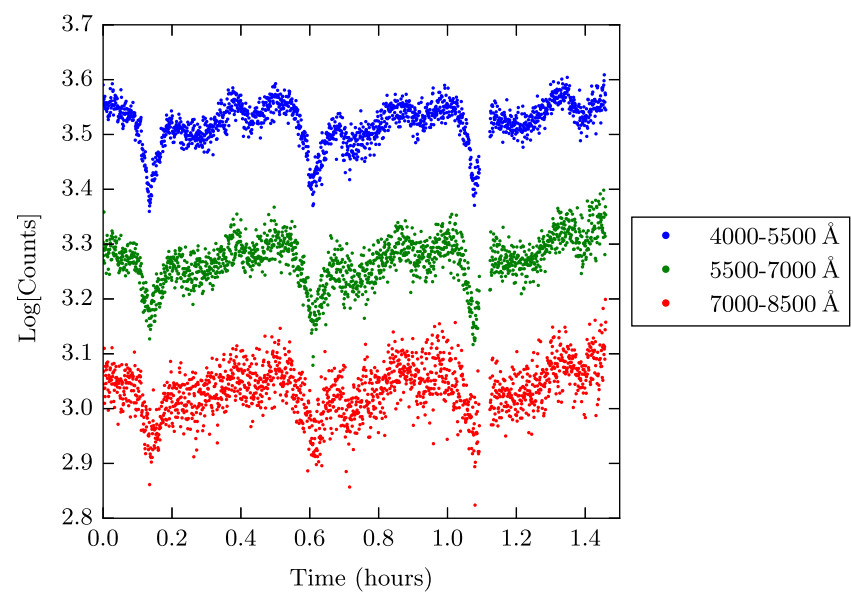

Figure 17. Set of light curves generated using simple Gaussian PSF fitting photometry on partially calibrated data in detector coordinate space, demonstrating the multi-wavelength capability of ARCONS. The photometry shows the eclipsing AM CVn star SDSS J0926+3624 in blue, green, and red bands (from top to bottom). Average standard deviations over a flat part of the light curve on short timescales are 118, 89, and 77 counts per $3 \mathrm{~s}$ integration, for the three wavelength bands, respectively. By comparison, the propagated PSF fitting errors are 138, 113, and 103 counts (larger likely because of systematic mismatch between the assumed Gaussian PSF model and the actual instrumental PSF). The shapes of the light curves are consistent with expectations, but we found no chromatic dependency, and the light curves match across the bands within our precision (see Szypryt et al. 2014).

and decl. positions. This is done by additionally binning the photons by wavelength, making a three-dimensional histogram of the R.A., decl., and wavelengths. The result is a data cube that can be summed across the spectral dimension to produce an image remapped onto a virtual grid in sky coordinates as 


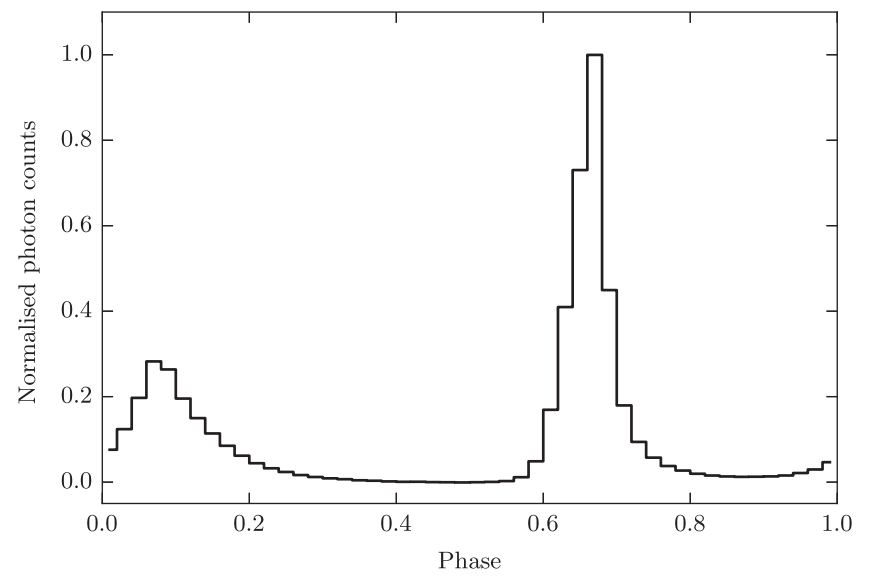

Figure 18. Pulse profile of the Crab Pulsar from aperture photometry on a fourdimensional "data hypercube" generated from fully calibrated photon lists in RA/decl. space, demonstrating ARCONS' high time resolution. Data are folded on the pulsar's $33 \mathrm{~ms}$ period to build adequate signal. The off-pulse level is taken to represent the background sky/nebulosity level, and is subtracted. Owing to the high integrated photon count, error bars are too small to be visible in this plot.

discussed in Section 5.1, or summed across chosen areas in R.A. and decl. to obtain a spectral energy distribution. Spectra are found using aperture spectroscopy, similar to standard aperture photometry, but with the photon counts per pixel binned by their respective wavelengths. The resulting total photon counts per wavelength of the object can then be converted to flux and plotted against wavelength to produce a spectrum. Figure 19 shows the results of aperture spectroscopy for a Crab Pulsar data cube.

By including an additional dimension in the histogram, it is also possible to include information about photon arrival time, making a four-dimensional histogram against R.A., decl., wavelength, and time. This four-dimensional data "hypercube" can be summed across any of its dimensions as desired, and sliced in order to obtain resolution in any dimension. This can be used to obtain the various products already described; it can also be used to obtain time-resolved spectra, for example. In the case of the Crab Pulsar, the emissions blink on and off very rapidly, with a period of $33 \mathrm{~ms}$. Figure 18 clearly shows that there are two main contributors to the overall flux coming from the object: the large main pulse, and the smaller inter-pulse. Since the Crab Pulsar is highly periodic, it is possible to bin the time axis by the pulsar's phase. After preparing a "hypercube" with dimensions of R.A., decl., wavelength, and pulsar phase, we can select photons where the pulsar is "off" and use that to make an estimate of the sky background spectrum within the photometric aperture itself, rather than using a sky annulus outside the aperture as an approximation, where nebulosity may affect the results. Similarly, it is possible to determine the spectrum for the main pulse and inter-pulse separately. The spectrum of the off-pulse can then be subtracted from the mainpulse and inter-pulse spectra at that same position for every virtual pixel within the aperture. The result is the sky-corrected spectrum of the main pulse and inter pulse. Figure 19 shows these phase-resolved spectra for the Crab Pulsar, showing good agreement with previously published data (Carramiñana et al. 2000; Sandberg \& Sollerman 2009). This technique demonstrates the full multi-dimensional capabilities of ARCONS, using imaging information to produce wavelength-resolved spectra with very fast time resolution.

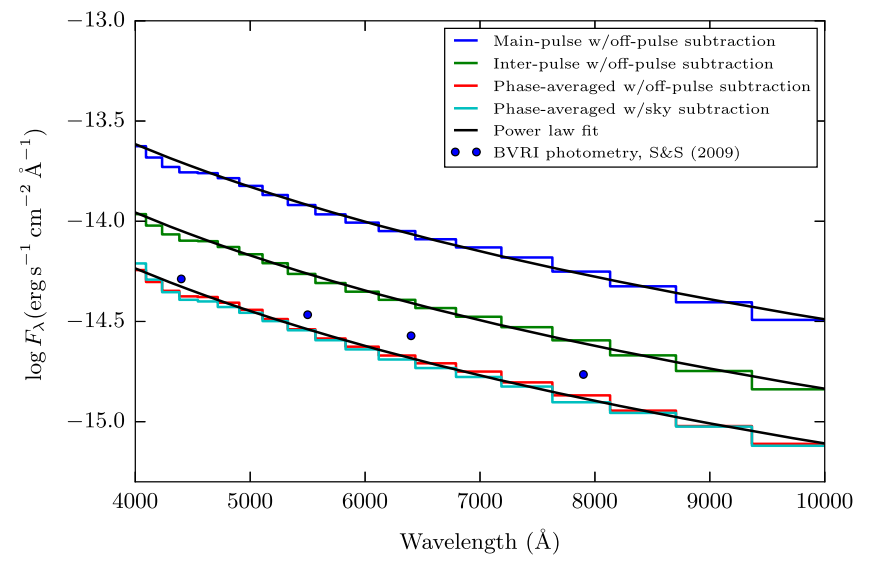

Figure 19. Spectra of the Crab Pulsar during the main-pulse, inter-pulse, and phase-averaged over the whole period, demonstrating the ability of ARCONS to perform time-resolved spectroscopy. All spectra are corrected for the effects of interstellar reddening according to $A_{\lambda} / E_{B-V}=-7.51 \log \left(\lambda / \lambda_{0}\right)+1.15$, with $\lambda_{0}=10000 \AA$ and $E_{B-V}=0.51$ (Carramiñana et al. 2000). The phaseaveraged spectra are integrated over all time, including the off-pulse time, resulting in a lower mean flux than the main- and inter-pulse spectra. The phase averaged spectrum generated by using the sky annulus subtraction is almost identical to that generated by using the off-pulse aperture subtraction. BVRI photometry points taken from Sandberg \& Sollerman (2009) are also dereddened and overplotted for comparison. The phase-averaged spectra follow the same trend as the photometry for all points, with the spectra falling within $\approx 10 \%$ of the BVRI photometry. This difference likely results from variations in flux from the Crab Pulsar and its surrounding nebula (Pacini 1971; Nasuti et al. 1996; Sandberg \& Sollerman 2009) and potential calibration errors for the ARCONS MKIDs array. The spectra of the main-pulse and inter-pulse are generated using the off-pulse aperture subtraction. Fits for the various spectra were found using a power law, $F_{\lambda}=K\left(\lambda / \lambda_{0}\right)^{-\alpha-2}$, with $\lambda_{0}=6000 \AA$ (per Carramiñana et al. 2000), and $4808 \AA<\lambda<9012 \AA$. The main-pulse and inter-pulse were found to have spectral indices of $\alpha_{\lambda}=+0.20$ and $\alpha_{\lambda}=+0.21$, respectively, agreeing with the values reported by Carraminana et al. (2000; $\alpha=+0.2 \pm 0.1$ for both pulses). The spectral index of the phase averaged spectrum was found to be $\alpha_{\lambda}=0.20$. Note that flux error estimates are too small to be appear in the plot (typically $\sim 10^{-4}$ for our data, and $\sim 10^{-3}$ for the BVRI points from Sandberg \& Sollerman 2009, in log space.)

\section{SUMMARY}

The pipeline as it currently stands takes the initial photon event detections from the instrument firmware, and passes them through the following main components: data calibration (cosmic-ray masking, bad-pixel masking, wavelength calibration, flatfielding, absolute spectral response calibration, astrometry, nonlinearity correction); calibrated photon list creation; and three output products, imaging, time-series photometry, and spectroscopy. The output products may all be based either on calibrated photon lists or on calibrated versions of the raw data files, depending on requirements and on how much field rotation and/or dithering or repointing has occurred over the total exposure time of interest.

Not all of these components are fully developed, and we expect much room for improvement as we begin to understand better the idiosyncrasies of the technology. Processing speeds are currently quite slow (on the order of a few times real-time), and as we move to larger array formats and increased observing time, performance will become an increasing concern. Translating time-consuming components to $\mathrm{C}$ should help, since native Python is not particularly fast. The massive data sets involved also lend themselves well to parallelization, which should yield significant speed gains. Such parallelization will be critical for high-speed applications such as speckle 
nulling (Martinache et al. 2014, S. R. Meeker et al., 2015 in preparation), where interference speckle noise in high-contrast coronagraphic images must be tracked on atmospheric seeing timescales $\left(\sim 10^{-2} \mathrm{~s}\right)$.

Four-dimensional image "hypercubes" (with dimensions of R.A., decl., wavelength, and time) remain to be fully implemented as an easily manageable final output product, though the elements to do so are largely in place. Other avenues for improvement to the pipeline include using the full wavelength- and time-resolved information available in the data to better characterize and discriminate hot pixels (to the extent that this is necessary with future generations of MKID arrays); fully integrating astrometric solutions from guide camera data rather than science camera data (to facilitate observing very faint objects); performing ex post facto image tip-tilt corrections for bright objects; improving our handling of photon-pileup and better understanding nonlinearity effects; and improved integration, streamlining, and automation of the pipeline.

MKID technology is still relatively young and rapidly developing. Our data reduction pipeline is therefore necessarily a work in progress, and is written with an eye to being readily adaptable to new and upgraded MKID arrays. The current ARCONS pipeline development code is made available at https://github.com/bmazin/ARCONS-pipeline.

We thank Shri Kulkarni and Tom Prince for the use of the Hale Telescope, and the staff of Palomar Observatory for their generous support. We are grateful to the anonymous referee for a careful and helpful review of our work. The MKID detectors used in this work were developed under NASA grant NNX11AD55G. The MKID digital readout was partially developed under NASA grant NNX10AF58G. S.R.M. and P.S. are supported by NASA Office of the Chief Technologists Space Technology Research Fellowships (NSTRF). J.C.v.E. was funded by a National Science Foundation ATI grant, number AST-1308556. This work was partially supported by the Keck Institute for Space Studies. Fermilab is operated by
Fermi Research Alliance, LLC under Contract No. De-AC0207CH11359 with the United States Department of Energy. STSCI_PYTHON is a product of the Space Telescope Science Institute, which is operated by AURA for NASA. This research made use of Astropy, a community-developed core Python package for Astronomy (Astropy Collaboration, et al. 2013). Based on observations obtained at the Hale Telescope, Palomar Observatory as part of a continuing collaboration between the California Institute of Technology, NASA/JPL, NOAO, Oxford University, Stony Brook University, and the National Astronomical Observatories of China.

Facility: Hale (ARCONS)

\section{REFERENCES}

Astropy Collaboration, Robitaille, T. P., Tollerud, E. J., et al. 2013, A\&A, 558, A33

Bertin, E., \& Arnouts, S. 1996, A\&AS, 117, 393

Carramiñana, A., Čadež, A., \& Zwitter, T. 2000, ApJ, 542, 974

Day, P. K., LeDuc, H. G., Mazin, B. A., Vayonakis, A., \& Zmuidzinas, J. 2003, Natur, 425, 817

Fruchter, A. S., \& Hook, R. N. 2002, PASP, 114, 144

Hébrard, G., Evans, T. M., Alonso, R., et al. 2011, A\&A, 533, A130

Hobbs, G. B., Edwards, R. T., \& Manchester, R. N. 2006, MNRAS, 369, 655

Hunter, J. D. 2007, CSE, 9, 90

Landolt, A. U. 1992, AJ, 104, 340

Magrath, B. 1997, PASP, 109, 303

Martinache, F., Guyon, O., Jovanovic, N., et al. 2014, PASP, 126, 565

Mazin, B. A., Bumble, B., Meeker, S. R., et al. 2012, OExpr, 20, 1503

Mazin, B. A., Meeker, S. R., Strader, M. J., et al. 2013, PASP, 125, 1348

McHugh, S., Mazin, B. A., Serfass, B., et al. 2012, RScI, 83, 044702

Nasuti, F. P., Mignani, R., Caraveo, P. A., \& Bignami, G. F. 1996, A\&A, 314,849

Oliphant, T. E. 2007, CSE, 9, 10

Pacini, F. 1971, ApJL, 163, L17

Sandberg, A., \& Sollerman, J. 2009, A\&A, 504, 525

Shupe, D. L., Moshir, M., Li, J., et al. 2005, in ASP Conf. Series, vol 347, Astronomical Data Analysis and System XIV, ed. P. L. Shopbell et al. (San Francisco, CA: ASP), 491

Strader, M. J., Johnson, M. D., Mazin, B. A., et al. 2013, ApJL, 779, L12

Szypryt, P., Duggan, G. E., Mazin, B. A., et al. 2014, MNRAS, 439, 2765

Yang, Z.-P., Ci, L., Bur, J. A., Lin, S.-Y., \& Ajayan, P. M. 2008, NanoL, 8,446 